\title{
Comparison of 4-Pole with 225 Coil-Turns and 6-Pole with 150 Coil-Turns Multi-pole Inner Coil Rotary MR Brake Designs
}

\author{
Ozgur Baser and Mehmet Alper Demiray
}

\begin{abstract}
Magneto-Rheological rotary brakes are widely used as passive torque actuators for stability in exoskeletons, humanoid robots, robot manipulators and haptic devices. An ideal MR brake should provide high braking torque performance with small outside dimensions. Among many MR brake designs in the literature, multi-pole design types have superior torque characteristics. Multi-pole MR brakes can be designed with different number of poles. Increasing the number of poles will improve the torque performance since it increases the number of magnetic circuit chains. On the other hand, this restricts the space required for the coils. Thus, the higher pole number necessitates less number of turn in each coil. In this paper, two design configurations of 4 -pole with 225 coil-turns and 6-pole with 150 coil-turns are selected for the same electrical resistance in terms of power consumption (4-pole $x$ 225 coil-turns $=6$-pole $\times 150$ coil-turns $=900$ coil-turns $)$. These two designs are analyzed and compared in the same outside dimensions $(27 \mathrm{~mm}$ radius $\times 85 \mathrm{~mm}$ length) by conducting analytical modelling, finite element analysis (FEA) and design optimization. The simulation results show that the 6-pole design configuration provides higher torque performance than 4-pole configuration although both have the same coil-turns in total.
\end{abstract}

Index Terms-Magneto-rheological fluid brake, multi-pole, rotary brake.

\section{AnAlytical Modelling}

Rotary Magneto-Rheological (MR) brakes presents braking torque based on MR fluid technology. In these devices, the fluid behaves like low viscosity oil while magnetic field doesn't exist and it has only off-state viscosity and Columb friction. When the MR fluid between rotor and housing part is exposed to magnetic field, the fluid's viscosity changes and high yield stress throughout the fluid occurs between rotor and housing. This is called as MR effect MR brakes have great advantages such as easy controllability, fast response time, low power requirement.

Basically, MR brakes are activated in three modes; shear mode, valve mode and pressure mode. In Fig. 1, operational concepts of these modes are given. In shear mode, magnetic field is applied to MR fluid which is located between a stationary part and a movable part. Magnetic flux lines lead to iron particles contained by the fluid to align like a chain and thus braking torque occurs by resisting the fluid flow. Rotary MR brakes commonly use shear mode. In the second mode,

Manuscript received February 5, 2016; revised June 13, 2016.

The authors are with the Department of Mechanical Engineering of Suleyman Demirel University, Isparta 32260, Turkey (corresponding author: Ozgur Baser; e-mail: ozgurbaser@sdu.edu.tr, mehmet.alper.demiray@gmail.com). called as valve mode, the parts are fixed and MR fluid between them is the movable. The fluid is enforced to move when the magnetic field is applied. Valve mode especially used in linear MR dampers. In the pressure mode, one part is fixed and the other part moves perpendicularly to compress the fluid.

Rotary MR brakes can be found with different structures in the literature and they were throughout investigated in some review papers [1], [2].
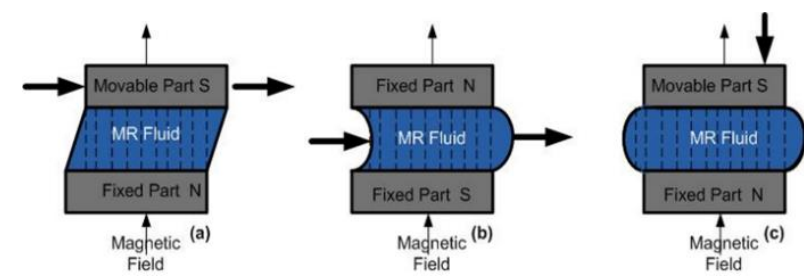

Fig. 1. Operational modes of MR brakes (a) shear mode (b) valve mode (c) pressure mode.

The main purpose of different designs is activating MR fluid more effectively and achieving the maximum torque in compact dimensions. Among the designs in literature, there is one promising design which is called as multi-pole coil structure proposed by Shiao et al. [3]. It provides high braking torque by means of the multiple magnetic line circuits activating MR fluid several times. Multi-pole coils are located in the outer part of the brake in the first design. Then, Shiao and Nguyen redesigned this multipole rotary MR brake design with adding some improvements [4]. This next study employs the multi-pole coil structure inside of the inner part of the rotary MR brake. Figs. 2 and 3 shows the inner coil multi-pole structure with its geometrical parameters. Multi-pole coils provide several magnetic flux circuits that can pass through MR fluid perpendicularly. Inner coil MR brake has larger rotor radius; therefore it produces higher braking torque with the same outside dimension.

In addition to the geometric parameters given in Fig. 2 and Fig. 3, another important parameter in multi-pole coil structure is the pole number. Normally, increasing pole number is useful to achieve higher braking torques due to the fact that a new pole means a new flux loop that can penetrate the fluid perpendicularly and so can activate new amount of the fluid. A comparison study was done by Shiao et al. for 4-pole, 6-pole and 10-pole MR brakes with the same winding turn number [5]. This study concluded that higher pole number provides higher braking torque. However, there can be a winding problem at this point as higher pole number limits the space required for the coils inside the brake. Especially for the compact brakes and limited volume 
applications, higher pole number limits the coil-turns number due to the lack of the space.

\begin{tabular}{ll} 
Parameters & \multicolumn{1}{c}{ Definition } \\
\hline Rotor Thickness & $: t_{r}=R_{2}-R_{1}$ \\
Rotor Radius & $: R_{r}=R_{2}$ \\
Stator Thickness & $: t_{s}=R_{5}-R_{4}$ \\
Stator Radius & $: R_{s}=R_{5}$ \\
MR Fluid Gap & $: g=R_{3}-R_{2}$ \\
Core Thickness & $: \mathrm{c}$ \\
Rotor Stator Width & $: \mathrm{z}$
\end{tabular}

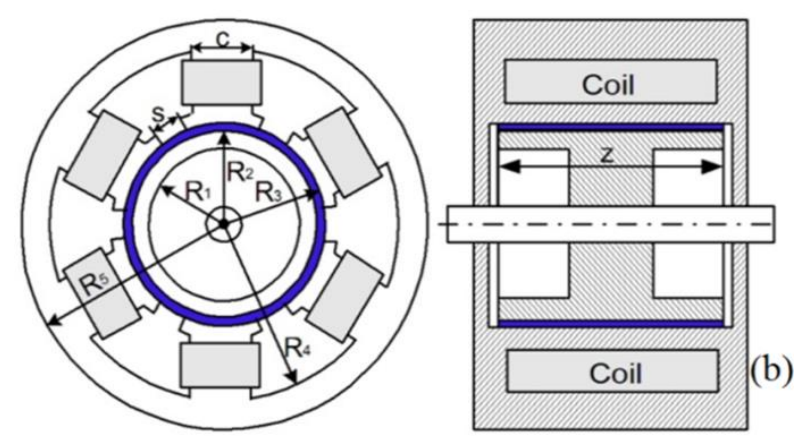

Fig. 2. Outer coil structured multi-pole MR brake (a) parameters and (b) schematic views.

\begin{tabular}{ll} 
Parameters & \multicolumn{1}{c}{ Definition } \\
\hline Rotor Thickness & $: t_{r}=R_{2}-R_{1}$ \\
Rotor Radius & $: R_{r}=R_{3}$ \\
Stator Thickness & $: t_{s}=R_{5}-R_{4}$ \\
Stator Radius & $: R_{s}=R_{5}$ \\
MR Fluid Gap & $: g=R_{4}-R_{3}$ \\
Core Thickness & $: \mathrm{c}$ \\
Rotor Stator Width & $: \mathrm{z}$
\end{tabular}

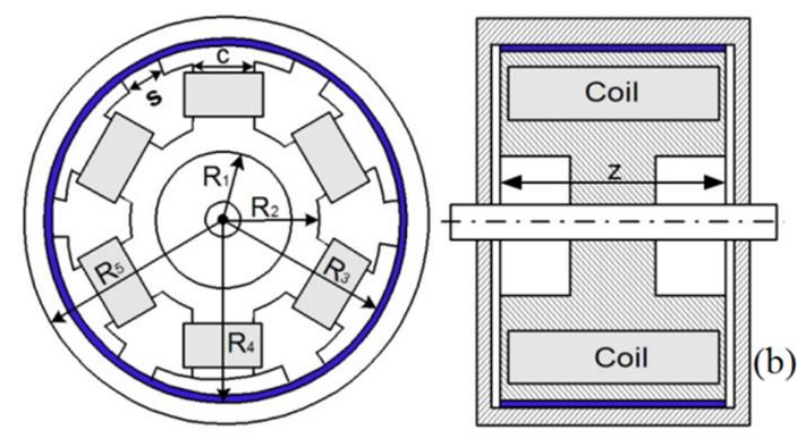

Fig. 3. Inner coil structured multi-pole MR brake (a) parameters and (b) schematic view.

For the reasons mentioned above, our study focuses on the torque output comparison of two types of inner coil multi-pole design configurations: 4-pole with 225 coil-turns and 6-pole with 150 coil-turns design configurations. These coil-turns numbers were selected for the same electrical resistance. Thus, these two configurations have totally 900 coil-turns which mean the same electrical resistance (4-pole $\times$ 225 coil-turns $=6$-pole $\times 150$ coil-turns $=900$ coil-turns $)$. Outer dimensions and the radius of the MR fluid circle were taken at the same values for two designs. The fixed geometric parameters of the 4-pole and 6-pole brake configurations are given in Table I.

TABLE I: The FiXed GeOMETRIC PARAMETERS OF THE 4-Pole AND 6-Pole DESIGN CONFIGURATIONS

\begin{tabular}{|l|c|c|c|c|}
\hline 4-pole (mm) & $z=70$ & $g=0.5$ & $R_{s}=27$ & $R_{r}=21.5$ \\
\hline 6-Pole (mm) & $z=70$ & $g=0.5$ & $R_{S}=27$ & $R_{r}=21.5$ \\
\hline
\end{tabular}

\section{ANALYTICAL MoDELling}

Yield strength of MR fluid is explained based on Bingham Plastic Model [6], [7] as below;

$$
\tau=\tau_{Y}+\eta \frac{r \omega}{g}
$$

$\tau_{Y}$ is the dynamic yield strength and second term on the right side is about fluid viscosity that contains radius $r$, angular velocity $\omega$, fluid gap $g$, and viscous friction term $\eta$. $\tau_{Y}$ term can be defined as a function of magnetic field strength of MR fluid $\left(H_{M R}\right)$. Among the MR fluid types from Lord Corporation's technical document [8], MRF-140CG fluid was selected for high magnetic field response, high yield stress in this study. Fig. 4 shows $\tau_{Y}$ of MRF-140CG fluid as a function of $H_{M R}$. By curve-fitting method, $\tau_{Y}$ can be formulated as a polynomial function of $H_{M R}$ on Fig. 4 and we obtained (2);

$$
\tau_{Y}=a_{1} H_{M R}^{3}+a_{2} H_{M R}^{2}+a_{3} H_{M R}+a_{4}
$$

where $a=-2.8 \times 10^{-29},-1.4 \times 10^{-9}, 5.9 \times 10^{-4}, 0.84$

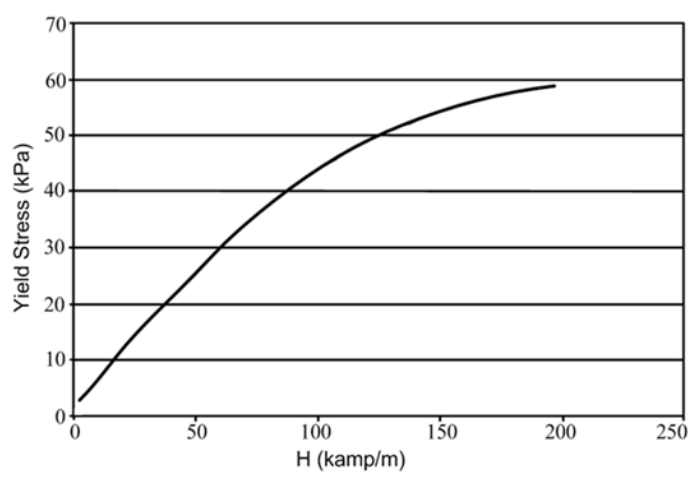

Fig. 4. MRF-140CG yield stress vs magnetic field strength [8].

Total MR brake torque is the combination of the field torque, the viscous torque and Coulomb friction torque components. The field torque component is the result of the surface integration of yield stress at the MR fluid gap area. These are formulated as given in (3) and (4) respectively.

$$
\begin{gathered}
T=T_{\text {field }}+T_{\text {viscous }}+T_{f r} \\
T_{\text {field }}=R_{M R}^{2} \int_{0}^{Z} \int_{0}^{2 \pi} \tau_{Y} d \theta d z
\end{gathered}
$$

$\theta, \mathrm{z}$ and $R_{M R}$ is the angular position, width and radius of the rotor of the MR brake, respectively. For precise MR brake torque calculations, finite element analysis (FEA) method is 
commonly used in literature. Therefore, we used ANSYS® Maxwell magnetic analysis program by employing (2) and (4) to calculate the field torque.

\section{SimULATION AND OPTIMIZATION}

In this section, magnetic FEA simulation was conducted to investigate the magnetic field density and magnetic field strength. The simulation results conducted on ANSYS $®$ Maxwell magnetic analysis program are given in Fig. 5 and Fig. 6 for two inner coil MR brake design configurations.

Some design parameters significantly affect the output braking torque. Therefore, these should be optimized before magnetic analysis. The design parameters that affect the braking torque are rotor thickness $\left(t_{r}\right)$, stator thickness $\left(t_{s}\right)$, core thickness (c), and slot (s). These all parameters are depicted in Fig. 2. Also, the intervals of these parameters for optimization were given in Table II.
TABLE II: THE INTERVALS OF THE DESIGN PARAMETERS NEED TO BE OPTIMIZED

\begin{tabular}{|c|l|}
\hline 4-Pole Intervals & 6-Pole Intervals \\
\hline $\min \left[t_{s}, t_{r}, s, c\right]=[3,3,5,5]$ & $\min \left[t_{s}, t_{r}, s, c\right]=[3,3,5,5]$ \\
\hline $\max \left[t_{s}, t_{r}, s, c\right]=[6,6,18,9]$ & $\max \left[t_{s}, t_{r}, s, c\right]=[6,6,9,9]$ \\
\hline
\end{tabular}

Optimetric Toolbox of ANSYS® Maxwell was used in the optimization. It employs Sequential Nonlinear Programing (SNLP) to find optimal values of the design parameters. The variation of the geometric parameters during 100 iterations is given in Fig. 7 and optimal values of the design parameters are in Table III.

TABLE III: OPTIMIZED VALUES OF THE DESIGN PARAMETERS

\begin{tabular}{|c|c|}
\hline $\begin{array}{c}\text { 4-Pole Optimal values of the } \\
\text { design parameters }\end{array}$ & $\begin{array}{c}\text { 6-Pole Optimal values of the } \\
\text { design parameters }\end{array}$ \\
\hline opt $\left[t_{s}, t_{r}, s, c\right]=[5.5,5,15,9]$ & $\operatorname{opt}\left[t_{s}, t_{r}, s, c\right]=[5.5,5.5,5.5,9]$ \\
\hline
\end{tabular}

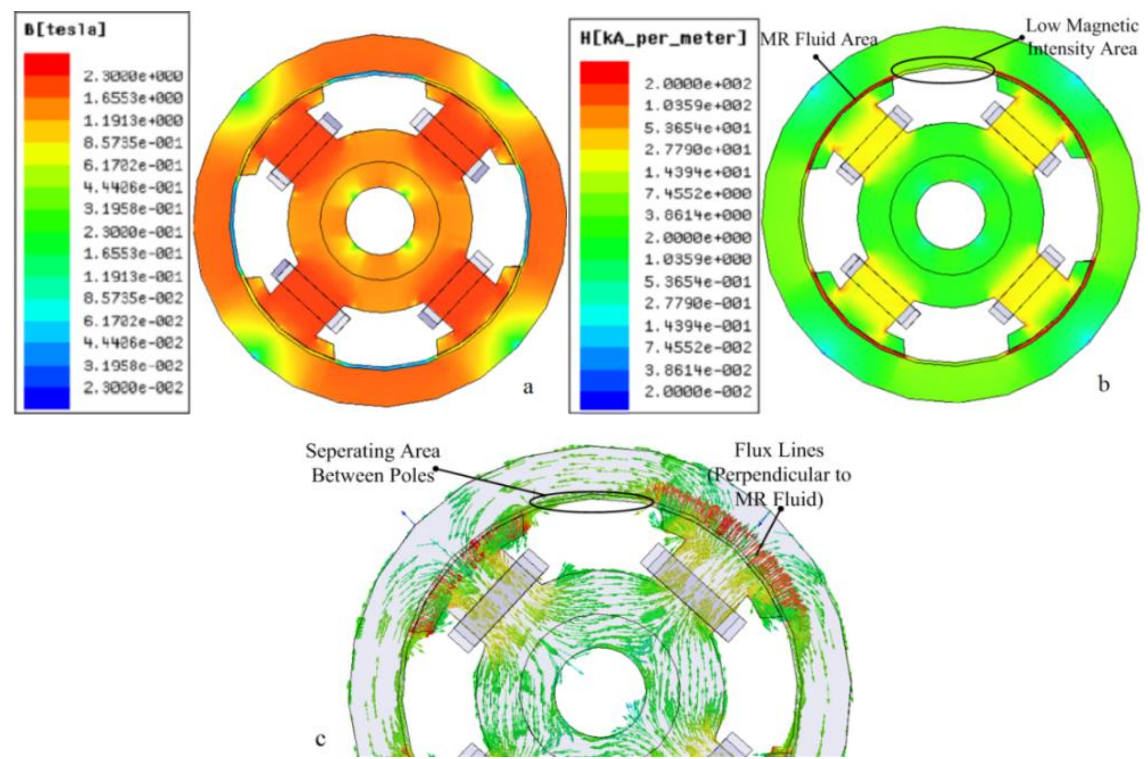

Fig. 5. (a) Magnetic field density, (b) Magnetic field strength and (c) Magnetic field vector for 4-pole MR brake.
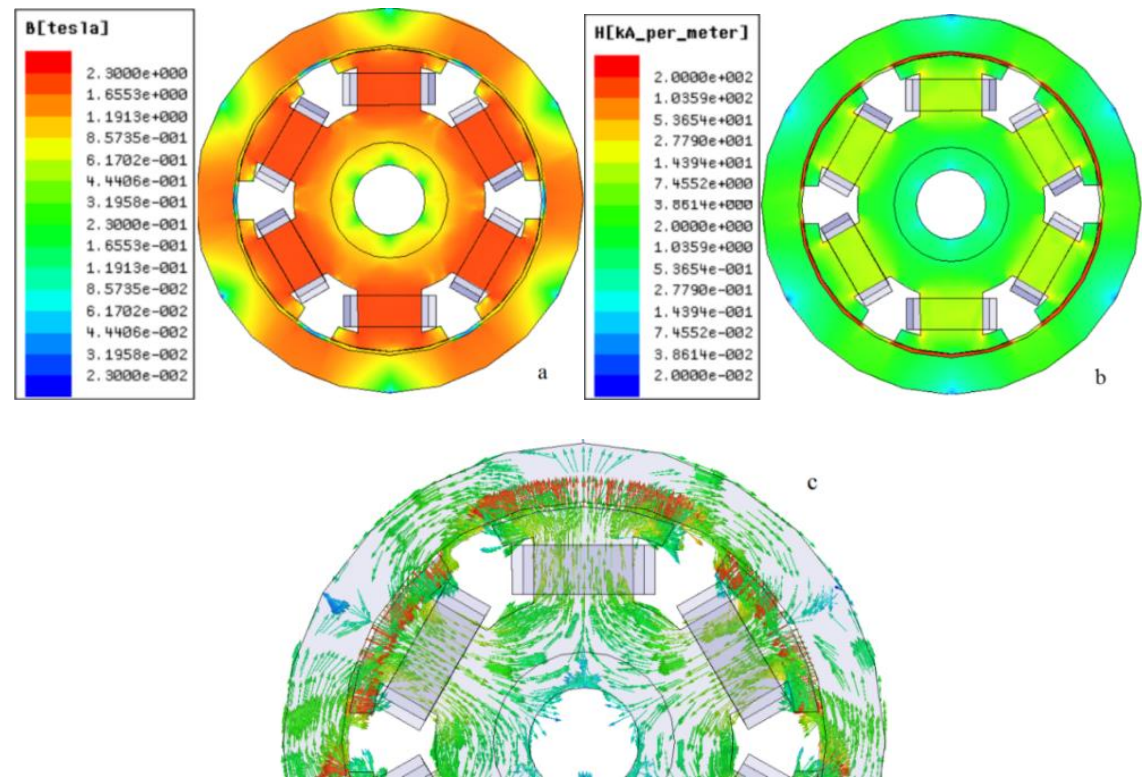

Fig. 6. (a) Magnetic field density, (b) magnetic field strength and (c) magnetic field vector for 6-pole MR brake.

After the optimization ended, magnetic colored maps of the 4-pole and 6-pole multi-pole MR brake designs were created at 1 Amper while the highest torque was generating. While Fig. 5a and Fig. 5b show the magnetic field density 
and magnetic field strength, Fig. 5c presents the direction of magnetic flux loops in vector form for 4-pole structured MR brake. Fig. 6 gives the same results for 6-pole MR brake. As can be seen from Fig. 5a and Fig. 5b, 4-pole structure is about saturated under the effect of 1 Amper current while 6-pole structured brake is away from the saturation at the same current level. Because, magneto motive force $(\mathrm{Ni})$ of 4-pole brake with 225 coil-turns is higher than the 6-pole brake with 150 coil-turns. In Fig. 5b and Fig. 6b, the MR fluid under pole head regions have the highest magnetic field because it is near of the core center. Fig. $5 \mathrm{c}$ and Fig. $6 \mathrm{c}$ prove that the magnetic field vectors penetrate the MR fluid perpendicularly around the pole heads and goes to the adjacent pole and close the magnetic field chains.

In addition to the magnetic field colored maps, the variation of the magnetic field strength $(\mathrm{H})$ was plotted on Fig. $8 \mathrm{a}$ and Fig. $8 \mathrm{~b}$ throughout the circumference of the MR fluid circle of the 4-pole and 6-pole MR brakes, respectively. Fig. 8a and Fig. 8b show that peak levels of the magnetic field strength are similar for the both designs although the coil-turns numbers were selected differently. However, 6-pole brake generates six peaks while it is four in 4-pole design. It means that 6-pole design has more activated MR fluid than the 4-pole design. Therefore, it is expected that the 6-pole with 150 coil-turns MR brake design provides higher braking torque than 4-pole with 225 coil-turns design. Finally, the field torque outputs of two MR brake designs were calculated by employing in ANSYS ${ }^{\circledR}$ Maxwell FEA program. The torque output results are shown in Fig. 9 as a function of supply current. According to Fig. 8, while 4-pole design gives $5.5 \mathrm{Nm}$ of maximum output torque, 6-pole design generated 7.5 of maximum output torque. As mentioned in the previous sections, two brakes were configured to provide same electrical coil resistance (4-pole $\times$ 225 coil-turns $=6$-pole $\times 150$ coil-turns $=900$ coil-turns) so that the both brakes consume the same power at the same supply current. In other words, 6-pole with 150 coil-turns design consumes less power than 4-pole with 225 coil-turns design for the same braking torque.
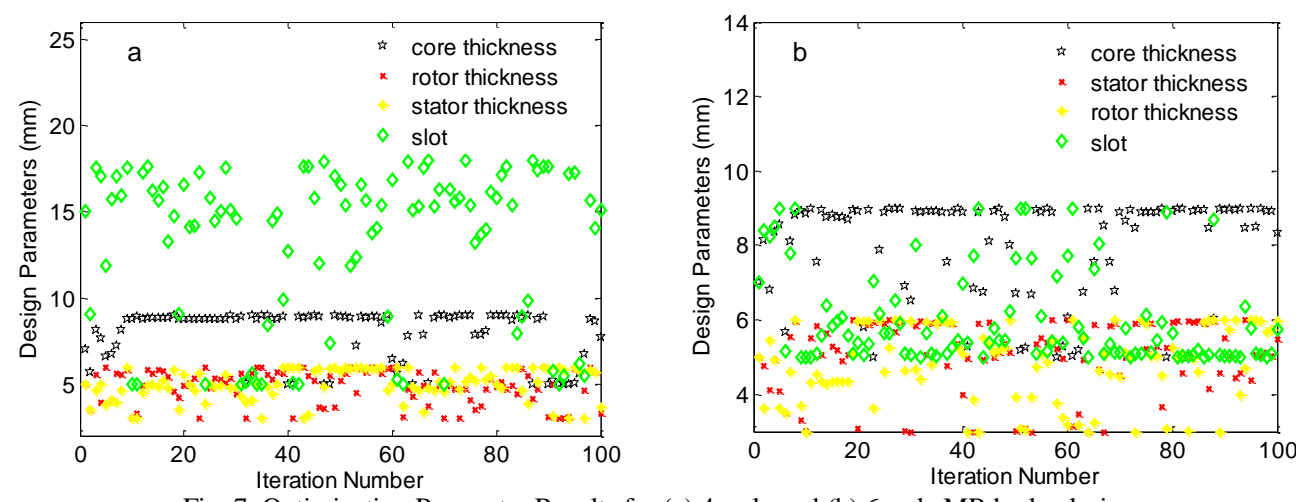

Fig. 7. Optimization Parameter Results for (a) 4-pole and (b) 6-pole MR brake designs.
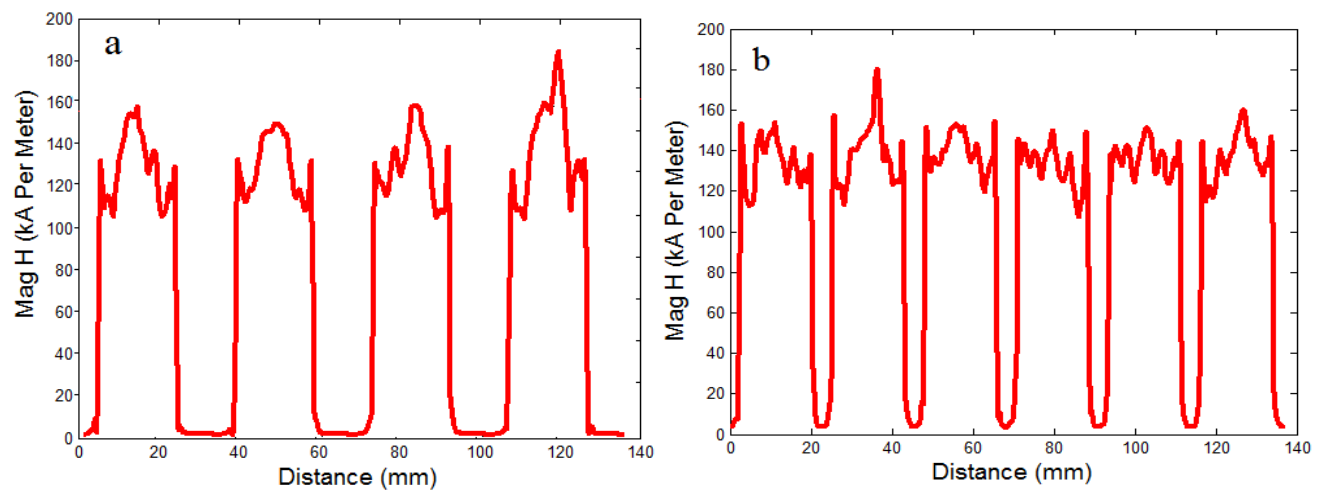

Fig. 8. Magnetic Field Strength Plots for (a) 4-pole and (b) 6-pole MR brake designs.

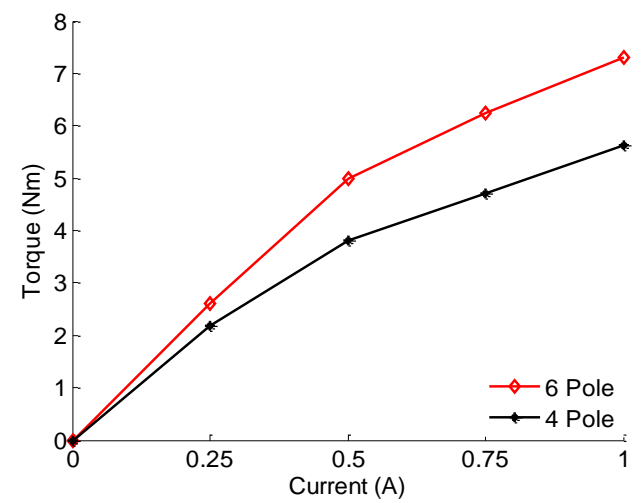

Fig. 9. Braking torque versus supply current for 4-pole with 225 coil-turns and 6-pole with 150 coil-turns MR brakes.

\section{CONCLUSION}

In this paper, 4-pole with 225 coil-turns and 6-pole with 150 coil-turns rotary MR brake designs are compared in the compact geometries. Each brake has the same outer dimensions and inner parameters. To provide the same total electrical coil resistance and to fit the wires in spaces between cores, coil-turns were selected different for two MR brake types $(4$-pole $\times 225$ coil-turns $=6$-pole $\times 150$ coil-turns $=900$ coil-turns). Both brakes were optimized and analyzed in ANSYS ${ }^{\circledR}$ Maxwell FEA program. After magnetic FEA and optimization process, it was shown that, 6-pole with 150 coil-turns and 4-pole with 225 coil-turns MR brake designs 
generate $7.5 \mathrm{Nm}$ and $5.5 \mathrm{Nm}$ of maximum torque with the same geometries, respectively. 6-pole with 150 coil-turns design type has higher torque capacity than the other 4-pole with 225 coil-turns design. However, assembling the 6-pole design is more complicated than the 4-pole design for compact sizes due to the wire wrapping difficulty inside the smaller space between the cores.

\section{ACKNOWLEDGEMENT}

The author would like thank to TUBITAK (The Scientific and Technological Research Council of Turkey) for the financial support of a research project numbered with 213 M297.

\section{REFERENCES}

[1] F. Imaduddin, S. A. Mazlan, and H. Zamzuri, "A design and modelling review of rotary magnetorheological damper," Materials and Design, vol. 51, pp. 575-91, 2013.

[2] Q. H. Nguyen and S. B. Choi, "Selection of magnetorheological brake types via optimal design considering maximum torque and constrained volume," Smart Materials and Structures, vol. 21, no. 1, p. 12, 2012.

[3] Y. Shiao and C. Y. Chang, "Design of an innovative high-torque brake," Advanced Material Research, vol. 339, pp. 84-87, Sep. 2011.

[4] Y. Shiao and Q. A. Nguyen, "Development of a multi-pole magnetorheological brake," Smart Materials and Structures, vol. 22, April 2013.
[5] Y. Shiao, Q. A. Nguyen, and Z. Zang, "Design and experiment of a new magnetoreological brake," International Journal of Applied Electromeagnetics and Mechanics, vol. 48, pp. 309-326, July 2015.

[6] Z. Leicht, H. Urreta et al., "Theoretical and experimental analysis of MR valve," Journal of Physics, Conference Series, vol. 149, no. 1, 2009.

[7] O. Ashour, C. A. Rogers, and W. Kordonsky, "Magnetorheological fluids: materials, characterization, and devices," Journal of Intelligent Materials, Systems and Structures, vol. 7, no. 2, pp. 123-130, March 1996.

[8] Lord Technical Data, MRF-140CG Magneto-Rheological Fluid, 2012.

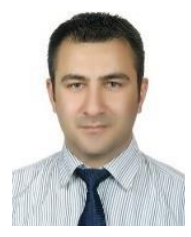

Ozgur Baser received his Ph.D. degree in the Department of Mechanical Engineering, Middle East Technical University, Turkey, in 2012. He is currently working at the Department of Mechanical Engineering, Suleyman Demirel University, Turkey. His research interests include robotics, exoskeletons, human-robot interaction, magnetorheological brakes.

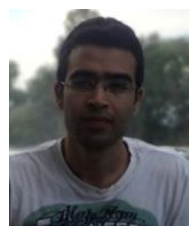

Mehmet Alper Demiray received B.S. Degree in Department of Mechanical Engineering of Suleyman Demirel University, Turkey, in 2014. He is currently studying on M.S. at the same department and university. His research interests include magnetorheological brakes and their applications on robots. 